\title{
Article \\ Conditional CRISPR-Cas Genome Editing in Drosophila to Generate Intestinal Tumors
}

\author{
Shivohum Bahuguna (D), Siamak Redhai *(D), Jun Zhou*D, Tianyu Wang, Fillip Port and Michael Boutros *(D)
}

check for updates

Citation: Bahuguna, S.; Redhai, S.; Zhou, J.; Wang, T.; Port, F.; Boutros, M. Conditional CRISPR-Cas Genome Editing in Drosophila to Generate Intestinal Tumors. Cells 2021, 10, 3156. https://doi.org/10.3390/cells10113156

Academic Editor: Cayetano González

Received: 27 September 2021

Accepted: 10 November 2021

Published: 13 November 2021

Publisher's Note: MDPI stays neutral with regard to jurisdictional claims in published maps and institutional affiliations.

Copyright: (c) 2021 by the authors. Licensee MDPI, Basel, Switzerland. This article is an open access article distributed under the terms and conditions of the Creative Commons Attribution (CC BY) license (https:// creativecommons.org/licenses/by/ $4.0 /)$.
German Cancer Research Center (DKFZ), Division Signaling and Functional Genomics, BioQuant and Medical Faculty Mannheim, Heidelberg University, D-69120 Heidelberg, Germany; s.bahuguna@dkfz-heidelberg.de (S.B.); tianyu.wang@dkfz-heidelberg.de (T.W.); f.port@dkfz.de (F.P.)

* Correspondence: siamak.redhai@dkfz-heidelberg.de (S.R.); j.zhou@dkfz.de (J.Z.); m.boutros@dkfz.de (M.B.)
Abstract: CRISPR-Cas has revolutionized genetics and extensive efforts have been made to enhance its editing efficiency by developing increasingly more elaborate tools. Here, we evaluate the CRISPRCas9 system in Drosophila melanogaster to assess its ability to induce stem cell-derived tumors in the intestine. We generated conditional tissue-specific CRISPR knockouts using different Cas9 expression vectors with guide RNAs targeting the BMP, Notch, and JNK pathways in intestinal progenitors such as stem cells (ISCs) and enteroblasts (EBs). Perturbing Notch and BMP signaling increased the proliferation of ISCs/EBs and resulted in the formation of intestinal tumors, albeit with different efficiencies. By assessing both the anterior and posterior regions of the midgut, we observed regional differences in ISC/EB proliferation and tumor formation upon mutagenesis. Surprisingly, high continuous expression of Cas9 in ISCs/EBs blocked age-dependent increase in ISCs/EBs proliferation and when combined with gRNAs targeting tumor suppressors, it prevented tumorigenesis. However, no such effects were seen when temporal parameters of Cas 9 were adjusted to regulate its expression levels or with a genetically modified version, which expresses Cas9 at lower levels, suggesting that fine-tuning Cas9 expression is essential to avoid deleterious effects. Our findings suggest that modifications to Cas9 expression results in differences in editing efficiency and careful considerations are required when choosing reagents for CRISPR-Cas9 mutagenesis studies. In summary, Drosophila can serve as a powerful model for context-dependent CRISPR-Cas based perturbations and to test genome-editing systems in vivo.

Keywords: CRISPR; Cas9; tumors; BMP; Notch; JNK; intestinal stem cells; aging

\section{Introduction}

The application of Clustered Regularly Interspersed Short Palindromic Repeats (CRISPR) - CRISPR-associated (Cas) systems for genomic studies has recently emerged as a powerful tool [1]. Initially discovered as a defense mechanism against invading viruses in bacteria and archaea, CRISPR-Cas has been adapted for genome engineering applications in many organisms [1,2]. In the most widely used CRISPR system, the RNA-guided endonuclease Cas9 is targeted to specific DNA sequences to create double-strand breaks (DSBs) by a short guide-RNA (gRNA) sequence containing several nucleotides that are complementary to the target gene of interest $[3,4]$. The only limitation in choosing a target site for the prototypical Cas9 from Streptococcus pyogenes is that it must be adjacent to a protospacer adjacent motif (PAM) site of the sequence NGG. Cleavage of the target site and subsequent DSBs are repaired by the cellular DNA repair pathways, which includes the error prone non-homologous end joining pathway (NHEJ pathway) and the more precise homology-directed repair (HDR). However, cellular repair pathways frequently lead to the insertion or deletion of nucleotides at the target site, often causing loss of function (LOF), or sometimes, gain of function mutations [5,6]. Indeed, in recent times, CRISPR-Cas9 has been used in various fields including cancer genetics [6]. 
Over the past century, Drosophila melanogaster has emerged as an important model organism for biological studies due in large part to the vast number of genetic tools developed in this organism [7,8]. Noticeably, the GAL4/UAS system has emerged at the forefront of these efforts [9]. GAL4 can bind to upstream activating sequence (UAS) and activate expression of downstream genes. By coupling GAL4 expression with defined tissue-specific promoters, UAS-driven genes can be restricted to a desired location in the fly. Additionally, temporal control of this system can be achieved by introducing the GAL80 ${ }^{\text {ts }}$ element, which binds the GAL4 transcriptional activation domain and prevents its activity at a permissive temperature of $18{ }^{\circ} \mathrm{C}$ [10]. However, at the restrictive temperature of $29^{\circ} \mathrm{C}$ or above, GAL80 ${ }^{\text {ts }}$ no longer binds and represses GAL4, therefore allowing UASdriven gene expression. Interestingly, CRISPR-Cas technology has been used in Drosophila melanogaster for heritable germline mutagenesis. By driving Cas 9 and gRNA with the binary GAL4/UAS system, biallelic mutations can be achieved in a tissue-specific manner [11-13].

Modifications to the CRISPR-Cas system in Drosophila have been made in the past to improve its editing efficiency [14]. Initially, both microinjection of Cas9 and single-guide RNAs (gRNA) in the form of either plasmids, in vitro transcribed RNA or ribonucleoprotein particles, and stable integration of DNA constructs into the genome were used for mutagenesis, albeit with different outcomes [15,16]. Moreover, editing rates were also observed to change when different integration sites of the CRISPR-Cas elements were used or by manipulating cis-regulatory elements $[17,18]$. Interestingly, it has been observed that very high expression levels of Cas9 resulting from some of the original expression constructs such as UAS-Cas9.P1 can be toxic to cells $[13,17]$. Attempts at reducing toxicity by expressing Cas9 from plasmids tuned to lower expression such as UAS-Cas9.P2 resulted in some success, but some toxicity remained [11]. More recently, by using upstream open reading frames (uORF) of different lengths, it has been possible to titrate Cas9 to more optimal levels, and constructs such as the UAS- $u^{M}$ Cas9 have been shown to be less toxic [11].

The intestinal tract of Drosophila melanogaster has been extensively used for studies into metabolism, regeneration, and cancer biology, and parallels can be drawn with mammalian systems due to its functional and anatomical similarities [19]. The Drosophila midgut is maintained by proliferating intestinal stem cells (ISCs) that give rise to differentiating enteroblast progenitors (EBs) that can further mature into absorptive enterocytes (ECs) or secretory enteroendocrine cells (EEs) [19]. Previous studies have highlighted numerous signaling pathways that are involved in aspects of ISC division, differentiation, and tissue damage. Of note, conserved pathways such as Notch and BMP/Dpp signaling have been reported to control various aspects of differentiation and proliferation [20]. To date, CRISPRCas9 studies in the Drosophila intestine have been limited [21,22]. Here, we performed an analysis of two popular Cas9 expression constructs (Cas9.P2 and $u^{M}$ Cas9) for their ability to specifically alter ISCs/EBs biology. We targeted the BMP, Notch, and JNK pathways with two gRNAs against Mothers against dpp (Mad), neuralized (neur), Notch, and hemipterous (hep) and assessed parameters such as ISC/EB number, mitotic index, tumor incidence, and morphological changes to the intestine. We found that targeting these pathways induces regional proliferation of stem cells and tumor formation, albeit to different extents. Importantly, continuous expression of Cas9.P2 in this tissue was deleterious to stem cells, but regulating Cas9.P2 expression with temporal control or using $u^{M}$ Cas 9 allowed efficient mutagenesis to take place.

\section{Material and Methods}

\subsection{Fly Stocks}

The following lines were used: esg-Gal4; tub-Gal80 ${ }^{\text {ts }}$, UAS-GFP (esg ${ }^{\text {ts }}$ ) [23], esg-Gal4, tub-Gal80 ${ }^{\text {ts }}$, UAS-GFP; UAS-Cas9.P2 (esg ${ }^{\text {ts }}>$ Cas9.P2) [11], esg-Gal4, tub-Gal80 ${ }^{\text {ts }}$, UAS-GFP; UAS- ${ }^{\mathrm{M}}$ Cas9 (esg $^{\text {ts }}>\mathrm{u}^{\mathrm{M}}$ Cas9) [11], P\{hsFLP $\} 1, \mathrm{y}^{1} \mathrm{w}^{1118} ; \mathrm{P}\{$ HD_CFD01184 $\}$ attP40-Notch gRNA $^{2 X}$ (VDRC 341922), P\{hsFLP $\} 1, y^{1} w^{1118} ;$ P\{HD_CFD00651 $\}$ attP40-Mad gRNA ${ }^{2 X}$ (VDRC 341570), P\{hsFLP $\} 1, \mathrm{y}^{1} \mathrm{w}^{1118}$; P\{HD_CFD01179\}attP40-neur gRNA ${ }^{2 X}$ (VDRC 341917), 
P $\{$ hsFLP $\} 1, \mathrm{y}^{1} \mathrm{w}^{1118} ; \mathrm{P}\left\{\mathrm{HD} \_C F D 01377\right\}$ attP40-hep gRNA ${ }^{2 \mathrm{X}}$ (VDRC 342022). All gRNA lines were previously made [11].

\subsection{CRISPR/Cas9 Mutagenesis}

Flies were kept on a standard cornmeal/agar diet with a $12 \mathrm{~h}: 12 \mathrm{~h}$ light:dark cycle. One L of standard diet contained $44 \mathrm{~g}$ sugar syrup, $80 \mathrm{~g}$ malt, $80 \mathrm{~g}$ corn flour premium G750, $10 \mathrm{~g}$ soy flour, $18 \mathrm{~g}$ yeast, $2.4 \mathrm{~g}$ methly-4-hyroxybenzoate, $6.6 \mathrm{~mL}$ propionic acid, $0.66 \mathrm{~mL}$ phosphoric acid, and $8 \mathrm{~g}$ agar [22]. Flies requiring adult expression of transgenes were initially raised at $18{ }^{\circ} \mathrm{C}$ (permissive temperature) and switched to $29^{\circ} \mathrm{C}$ (restrictive temperature) post eclosion for specific durations. For experiments involving UAS-Cas9.P2, newly eclosed flies were shifted to $29^{\circ} \mathrm{C}$ for 10 days and $18{ }^{\circ} \mathrm{C}$ for 30 days before being switched back to $29^{\circ} \mathrm{C}$ for one day. For experiments involving $U A S-u^{M} \mathrm{Cas} 9$, newly eclosed flies were shifted to $29^{\circ} \mathrm{C}$ for 20 days. Flies were transferred into fresh food once every two days to avoid fungal infection. Mated females were used for all experiments.

\subsection{Immunostaining and Image Acquisition}

Adult flies were anesthetized on a $\mathrm{CO}_{2}$ pad and female flies were dissected in $1 \times$ PBS. Whole guts were transferred onto poly-lysine slides and fixed with $4 \%$ formaldehylde (28908, VWR diluted in PBS) for $30 \mathrm{~min}$. The intestines were then washed with $1 \times$ PBST $(1 \times$ PBS $+0.1 \%$ Triton X-100 (T8787-250 mL, Sigma, Darmstadt, Germany)) and blocked with $1 \times$ PBST $+1 \%$ BSA (1062, Gerbu, Heidelberg, Germany). The tissues were incubated overnight with the primary antibody at $4{ }^{\circ} \mathrm{C}$ and washed with $1 \times$ PBST. Fluorescently labelled secondary antibodies were diluted in $1 \times$ PBST (final concentration 1:3000) and incubated at room temperature for $1.5 \mathrm{~h}$. The midguts were then mounted in Vectashield containing DAPI (H-1200, Linaris, Burlingame, CA, USA). Staining of experimental and control samples was carried out on the same slide for direct comparisons. The same confocal settings (e.g., laser power, gain, and pinhole) were applied to both experimental and control groups. Primary antibodies were rabbit anti-pH3 (1:500, cell signaling 9701L), mouse anti-Cas9 (1:500 Cell signaling 14,697 s), and secondary antibodies were chicken anti rabbit AF594 (1:3000, A21442, Invitrogen, Waltham, MA, USA) and donkey anti mouse AF594 (1:3000, A21203, Invitrogen, Waltham, MA, USA).

\subsection{Quantification of Midgut Mitosis}

Dividing cells were marked with Phospho-Histone H3 staining (pH3) antibody and mitotic cells were counted in the anterior and posterior intestine using a Leica SP8 confocal microscope with a $40 \times$ objective. Representative images were taken using a Leica SP8 confocal microscope. Images were analyzed, processed, and compiled using Prism 8, Fiji [24], and Inkscape.

\subsection{Quantification of GFP Positive Cells}

Whole z-stacked images of the entire anterior (R2) and posterior (R4 and R5) midgut regions were taken using either a Leica SP5, SP8, or a Nikon A1 confocal microscope. The progenitor cells labelled with GFP (esg-GAL4, tub-Gal80 $\left.{ }^{t s}, U A S-G F P\right)$ and nuclei of all intestinal cells labelled with DAPI were counted using an ImageJ macro (developed by Dr Damir Krunic from the DKFZ imaging facility). This function segments the intestines and counts the total number of $\mathrm{DAPI}^{+}$nuclei and $\mathrm{GFP}^{+}$cells. We used the following formula: $\left(\mathrm{GFP}^{+} / \mathrm{DAPI}^{+}\right) \times 100$, to generate the percentage of $\mathrm{GFP}^{+}$cells in the region of interest. Images were analyzed, processed, and compiled using Prism 8, Fiji [24], and Inkscape.

\subsection{Measurement of Midgut Length and Diameter}

Guts were dissected, placed on a polylysine-coated slide and fixed with $4 \%$ formaldehyde (28908, VWR diluted in PBS) for $30 \mathrm{~min}$. Images of the entire midgut were taken using a Zeiss stereo microscope. Lines were drawn from the base of the proventriculus 
until the midgut-hindgut boundary using the free hand line tool in Fiji. The length of lines was measured and plotted in Prism 8. Full length representative images of different genotypes were taken using a Leica SP8 microscope on a $20 \times$ objective lens and stitched together using the pairwise stitching tool from Fiji. For gut diameter measurements, a z-projected view of the intestine was used, and a line was drawn perpendicular to the gut and measured with Fiji. Images were compiled in Inkscape.

\subsection{Tumor Incidence}

The tumor incidence was determined by counting the number of midguts with tumors in the mutant and control group. Tumors were defined as clusters of GFP positive cells with small DAPI nuclei. Representative images are shown for what we considered a tumor in Figure 4A. The percentage of tumor bearing flies were obtained from 2-3 independent experiments and the results were pooled to generate the related bar figure.

\subsection{Statistical Analysis}

All data were analyzed using Prism 8 software (GraphPad Software, San Diego, CA, USA). For comparisons between two groups, a student $t$ test was performed and for more than two groups, an ordinary one-way ANOVA test was used with multiple comparisons between groups. An outlier test was performed on datasets to remove any outliers. Significance values are represented as follows: ns: not significant, ${ }^{*} p<0.05$, ${ }^{* *} p<0.01,{ }^{* * *} p<0.001,{ }^{* * * *} p<0.0001$. All data points represent one animal and are marked with individual dots, data are shown using either a heatmap, stacked bar charts, or boxplots.

\section{Results}

\subsection{Cas9 Expression in the Drosophila Midgut}

The Drosophila midgut is compartmentalized and comprises several cell types including ISCs that can divide and give rise to EBs, which further differentiate into secretory enteroendocrine cells or absorptive enterocytes (Figure 1A). We used the esgGal4, tub$\mathrm{Gal}^{t s}$, UAS-GFP $\left(\mathrm{esg}^{\mathrm{ts}}\right)$ system to target progenitor cells including intestinal stem cells and enteroblasts (ISCs/EBs) in adult flies, circumventing defects that might arise due to the expression of genetic constructs during development (see Materials and Methods). We first crossed two Cas9 constructs (Cas9.P2 and $u^{M}$ Cas 9 ) into the esg ${ }^{t s}$ fly in order to stably express Cas 9 during adulthood using temporal control (esg g ${ }^{t s}>$ Cas9.P2 or esg $g^{t s}>u^{M}$ Cas 9 ). While Cas9.P2 has previously been shown to have strong expression, $u^{M}$ Cas 9 harbors an upstream opening reading frame, which dampens its expression, thus allowing us to perform mutagenesis in different conditions. To assess whether our esg ${ }^{t s}$ system expresses Cas9 specifically in progenitor cells, we induced its expression and performed antibody staining against Cas9. We found that in flies expressing esg ${ }^{t s}>$ Cas9.P2, Cas9 staining was detectable in ISCs/EBs, albeit with different degrees, which likely arises as a result of variable Gal4 expression (Figure 1B). In flies expressing es $g^{t s}>u^{M}$ Cas9, Cas9 was not detectable by immunostaining in ISCs/EBs, supporting the model that $u^{M}$ Cas 9 is expressed at a lower level compared to Cas9.P2 (data not shown). In our previous study, we showed that Cas9 immunostaining is detectable with $u^{M}$ Cas9, but at a lower level when compared to Cas9.P2 in the wing disc [11]. In summary, high expression of Cas9 in ISCs/EBs resulted in detectable Cas9 protein in these cell types. 
A

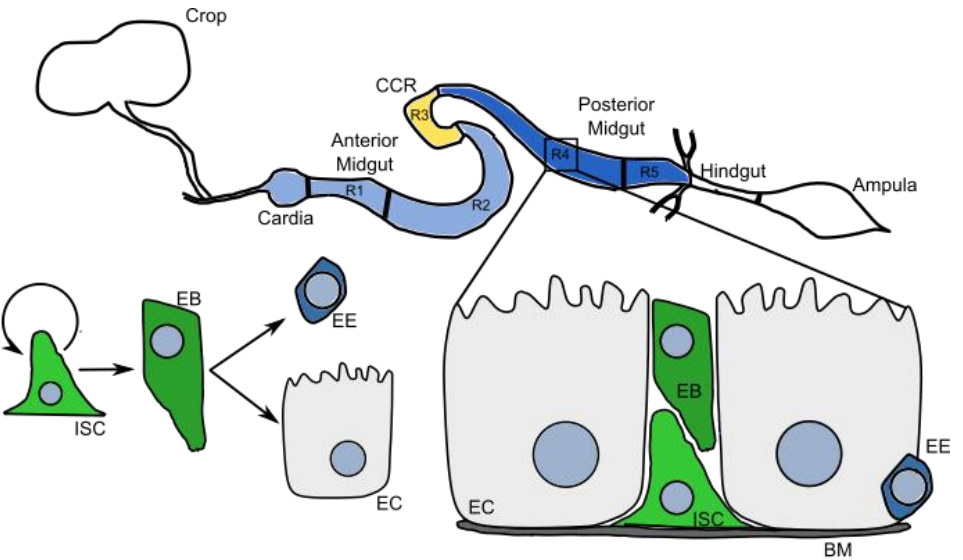

B

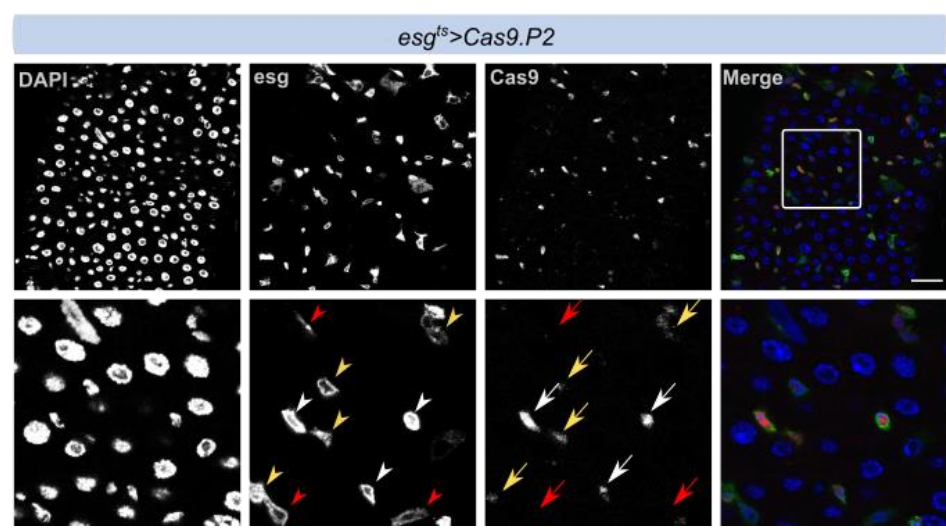

Figure 1. Digestive tract and intestinal cell types in Drosophila melanogaster. (A) Schematic representation of the intestine, which contains different regions (R1-R5) that house progenitor cells such as intestinal stem cells (ISCs) and enteroblasts (EBs), which can differentiate into enterocytes (ECs) or enteroendocrine cells (EEs). (B) ISCs/EBs are dispersed throughout the midgut and can be marked with escargot (esg > GFP) (arrowheads). Notice that esg $>$ GFP expression is variable (compare white and yellow arrowheads). Expressing Cas9.P2 using the esg $g^{t s}$ system and staining against Cas 9 (Red) reveals that this construct is translated into protein in ISCs/EBs (arrows). Notice that Cas9 expression is variable (compare white and yellow arrows) and some esg $>$ GFP cells lack detectable Cas9 expression (red arrowheads and red arrows). Scale bar: $30 \mu \mathrm{m}$.

\subsection{Defining Temporal Parameters for Cas9 Editing}

To test if continuous Cas9 expression is deleterious to ISCs/EBs, we expressed both Cas 9 constructs for different durations and quantified ISCs/EBs numbers. Previous reports suggest that the number of ISCs/EBs increases with age [25]. We used two different timepoints (seven days and 20 days) to assess whether ISCs/EBs numbers were altered in the R4 region of the intestine when different Cas 9 transgenes were expressed. In the control animals, we observed a significant increase in ISCs/EBs from seven days to 20 days (Figure 2A-C). Although continuous expression of Cas9.P2 did not alter the number of ISC/EBs at seven days (Figure 2A,C), it did significantly reduce their numbers at 20 days, suggesting that prolonged expression of Cas $9 . P 2$ may be deleterious to these cells (Figure 2A-C). We also observed that continuous expression of Cas9.P2 caused ISCs/EBs to appear more rounded (Figure 2B). Interestingly, no such effects were observed for $u^{M}$ Cas 9 , neither at seven days nor 20 days, and ISCs/EBs increased in an age-dependent manner similar to the control animals, suggesting that this construct is well tolerated in the intestine (Figure 2A-C). 
A

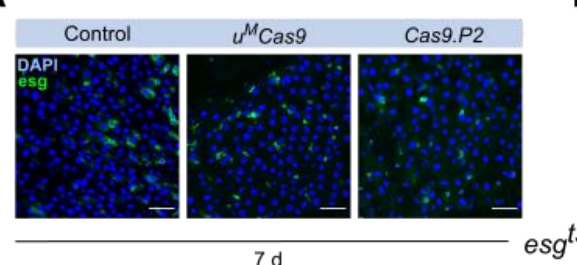

B
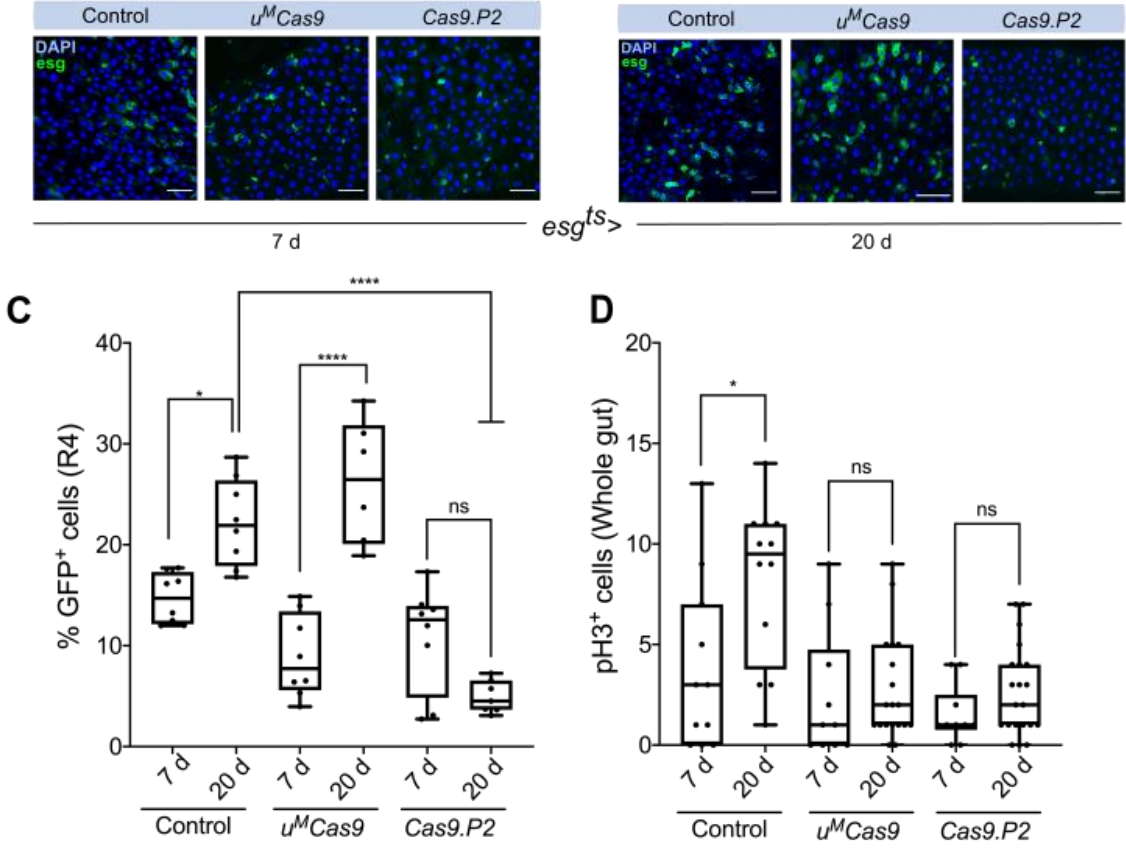

D

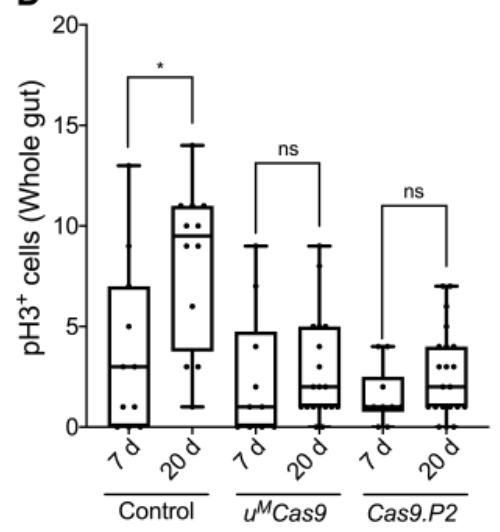

$e s g^{t s}>$

Figure 2. Continuous expression of Cas9.P2 is deleterious to ISCs/EBs. (A) ISCs/EBs in control animals displayed an age-dependent increase in their numbers (A; 7 days, B; 20 days, see quantifications in panel C). (B) Continuous expression of Cas9.P2, but not $u^{M}$ Cas9, for 20 days noticeably reduced ISCs/EBs. (C) Quantifications of ISCs/EBs numbers at seven days and 20 days in animals either expressing no Cas 9 , $u^{M}$ Cas9, or Cas9.P2. Notice that in both the control animals and those expressing $u^{M}$ Cas9, ISCs/EBs significantly increased from seven days to 20 days. (D) The number of mitotic cells increased in the whole gut of the control animals from seven days to 20 days, but this could not be seen in animals continuously expressing Cas9.P2 at these timepoints. Scale bar: $30 \mu \mathrm{m} .{ }^{*} p<0.05,{ }^{* * * *} p<0.0001$.

To complement these results, we performed the same time-course experiments in the control and esg $g^{t s}>$ Cas9.P2 flies and stained the intestines with phospho-histone 3 (pH3), which marks mitotically active cells. Whilst mitotic cells in control flies increased from seven days to 20 days, this effect was abrogated in flies continuously expressing Cas9.P2 for 20 days (Figure 2D). Surprisingly, we did not observe an increase in the number of mitotic cells in flies expressing esg ${ }^{t s}>u^{M}$ Cas 9 during these timepoints, suggesting that $u^{M}$ Cas 9 also has some deleterious effects on actively dividing cells. In conclusion, continuous expression of Cas9.P2 prevents an increase in ISC/EB and $\mathrm{pH} 3^{+}$cells, while expressing $u^{M}$ Cas 9 led to an age-dependent increase in ISCs/EBs but not $\mathrm{pH}^{+}$cells.

\subsection{Cas9.P2 ${ }^{\text {on/off }}$ and $u^{M}$ Cas $9^{\text {on }}$ Are Effective at Inducing Intestinal Tumors}

We set out to test the editing efficiencies of Cas9.P2 and $u^{M} C a s 9$ by targeting members of several conserved signaling pathways known for their role in tumor development. These include the BMP, Notch, and JNK cascades [21,26,27]. We assessed both the anterior and posterior portions of the intestine to test for regional differences in the number of ISC/EB derived tumors. Since continuous expression of Cas9.P2 is toxic to ISCs/EBs, we designed a temperature shift regiment to tune its expression and reduce toxicity. We found that switching Cas9.P2 expression on for 10 days and then off for 30 days was sufficient, allowing for efficient editing to take place (Figure $3 \mathrm{~A}, \mathrm{~B}$ ). At the end of this timepoint, we switched the system on for one day in order to express GFP and visualize ISCs/EBs

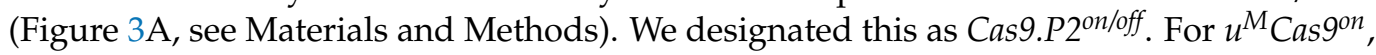
we found 20 days of expression to be sufficient for mutagenesis and also included Cas9.P2 on at this timepoint for direct comparison (Figure 3A,B). 


\section{A}

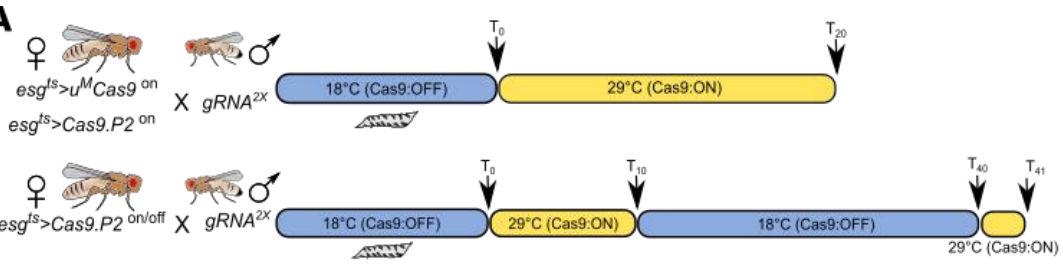

B

C
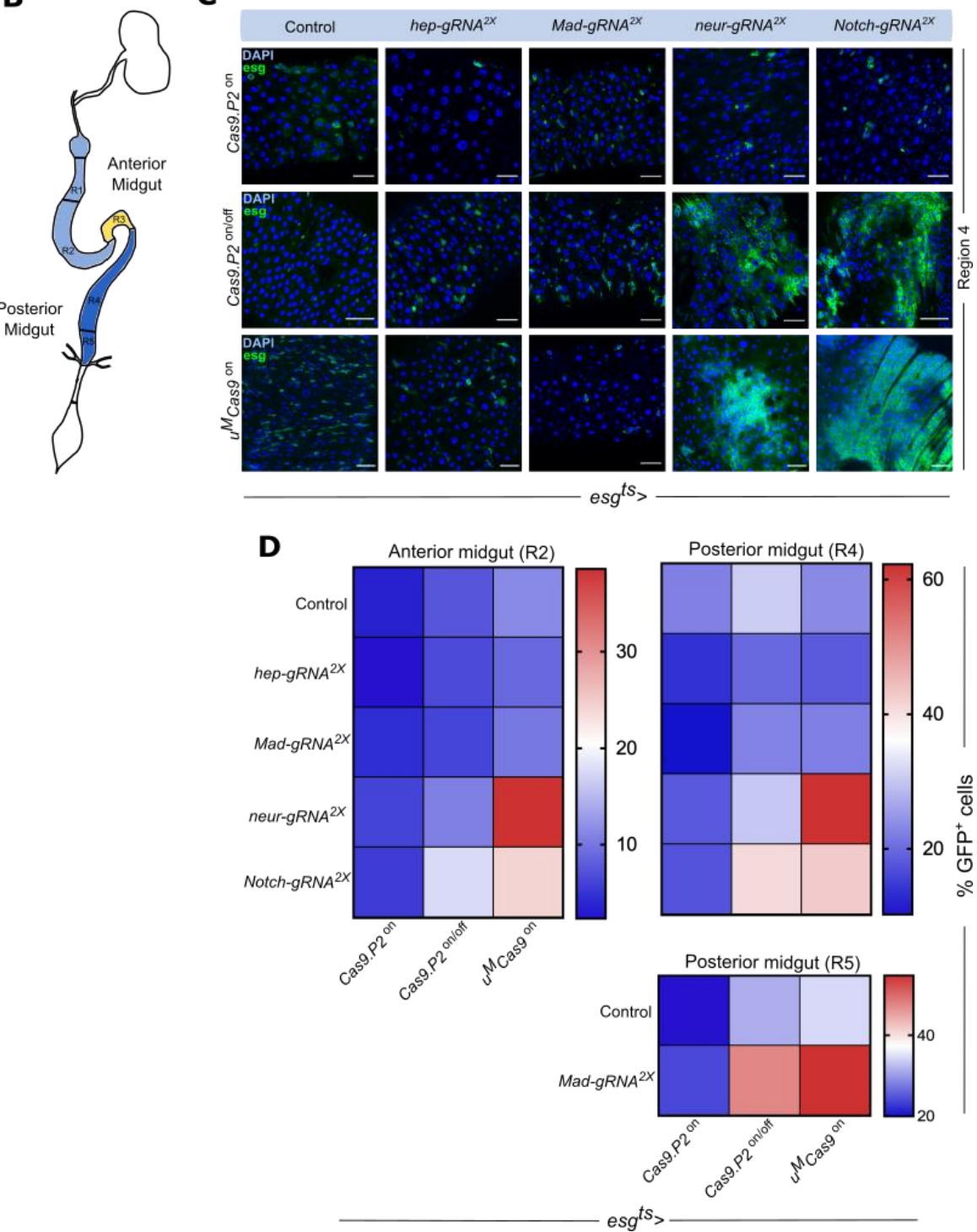

Figure 3. Both Cas9.P2 $2^{\text {on }}$ off and $\mathrm{u}^{\mathrm{M}} \mathrm{Cas} 9^{\text {on }}$ are effective at inducing intestinal stem cell derived tumors. (A) Schematic representation of the timepoints used for experiments. Female flies expressing either Cas9.P2 ${ }^{o n}$ or $u^{M} C a s 9^{o n}$ were crossed to male flies expressing different $g R N A s$. The cross was maintained at $18^{\circ} \mathrm{C}$ and the progeny were raised under these conditions during their development. After adult progeny emerged $\left(\mathrm{T}_{0}\right)$, they were shifted to $29^{\circ} \mathrm{C}$ continuously for 20 days to induce the esg $g^{t s}$ expression system, which allows for both Cas 9 and $g R N A s$ to be expressed in ISCs/EBs. Since high expression of Cas9.P2 is toxic, we also devised an on/off strategy (Cas9.P2 ${ }^{\text {on/off }}$ ), whereby adult progeny were initially shifted to $29^{\circ} \mathrm{C}$ for 10 days $\left(T_{10}\right)$, then to $18{ }^{\circ} \mathrm{C}$ for 30 days $\left(\mathrm{T}_{40}\right)$ and finally at $29^{\circ} \mathrm{C}$ for one day $\left(\mathrm{T}_{41}\right)$. (B) Schematic representation of the intestine with different regions highlighted. (C) Confocal images of the R4 regions of the intestine in flies expressing Cas 9 and $g R N A s$ against hep, Mad, neur, and Notch. Notice that for Cas9.P2 ${ }^{\text {on } / \text { off }}$ and $u^{M}$ Cas gon with $g R N A$ against neur and Notch resulted in massive proliferation of ISCs/EBs. (D) Heatmap showing the average percentage of $\mathrm{GFP}^{+}$ISCs/EBs after using different Cas9 enzymes for perturbations. Scale bar: $30 \mu \mathrm{m}$. 
We next used two transgenic $g R N A s\left(g R N A^{2 x}\right)$ from the Heidelberg CRISPR Fly Design Library [11] to target the tumor suppressors Mad, Notch, and neur with either Cas9.P2 (esg $g^{t s}>$ Cas9.P2) or $u^{M}$ Cas9 (esg $g^{t s}>u^{M}$ Cas9) and dissected the intestine of the progeny at the timepoints above-mentioned (Figure $3 \mathrm{~A}$ ). We also used $g R N A^{2 x}$ against hep as a control since mutating this gene has previously been shown to inhibit age-dependent increase of ISCs/EBs [25]. Continuous expression of Cas9.P2 ( $\left.\mathrm{esg}^{t s}>\mathrm{Cas} 9 . \mathrm{P}^{\mathrm{on}}\right)$ failed to show observable changes in ISC/EB numbers when perturbing either Mad, Notch, or neur, suggesting either low-efficiency mutagenesis or suppression of proliferation normally associated with such mutations in these genes by Cas 9 mediated toxicity (Figures $3 \mathrm{C}, \mathrm{D}$ and S1A). However, adopting an on/off regiment for Cas9.P2 (esg $g^{t s}>$ Cas9.P2 $2^{\text {on }}$ off $)$ increased ISC/EB numbers in the R4 region when either Notch or neur was perturbed and decreased ISCs/EBs numbers when using $g R N A^{2 x}$ against hep (Figures $3 \mathrm{C}, \mathrm{D}$ and S1B). Moreover, perturbations of Notch with Cas9.P2 ${ }^{\text {on/off }}$ also increased ISC/EB numbers in the R2 region, highlighting regional differences when compared to neur (Figures 3D and S1B). These changes have been previously observed in LOF studies of these genes, confirming the specificity of the gRNAs used [22,26,27]. Interestingly, $g R N A^{2 x}$ against $M a d$ failed to increase ISC/EB proliferation in the R2/R4 region, but did increase their numbers in the R5 region, highlighting regional specificity (Figures $3 \mathrm{D}$ and $\mathrm{S} 2 \mathrm{~A}, \mathrm{~B}$ ). In flies continuously expressing $u^{M}$ Cas 9 $\left(\right.$ esg $g^{t s}>u^{M}$ Cas $\left.9^{o n}\right)$ with $g R N A^{2 x}$ targeting neur and Notch, we observed a significant increase in ISC/EB proliferation in both R2/R4 regions, although the effect for Notch in the R2 region was more modest when compared to neur (Figures 3C,D and S1C). Similar to Cas9.P2 ${ }^{\text {on/off, }}$ $u^{M} C a s 9^{o n}$ showed regionalized proliferation of ISCs/EBs in the R5 region after mutating Mad (Figures 3D and S2A,B), although this construct did not change ISC/EB numbers when $g R N A^{2 x}$ against hep was used (Figure 3C,D). In summary, both $u^{M}$ Cas $9^{\circ n}$ and Cas9.P2 ${ }^{\text {on } / o f f}$ are effective tools to induce mutations in the intestine and for studying tumorigenesis.

\subsection{Mutating Tumor Suppressors Increases Mitotic Cells and Tumor Incidence}

To complement the findings above, we assayed mitotic activity by staining the intestine with $\mathrm{pH} 3$, which marks mitotic cells. Both Cas9.P2 ${ }^{\text {on } / \text { off }}$ and $u^{M} \mathrm{Cas} 9^{\text {on }}$, but not Cas9.P2 ${ }^{\text {on }}$, increased the number of mitotic cells after targeting either neur, Notch, or Mad (Figures S3C,D and S4A-C). Indeed, we also observed regional changes in $\mathrm{pH} 3$ numbers, with perturbations of Mad resulting in higher $\mathrm{pH}^{+}$cells in the posterior $\mathrm{R} 5$ portion of the intestine compared to the anterior (Figures S3B,C and S4B,C). When targeting Notch or neur with either Cas9.P2 ${ }^{\text {on/off }}$ and $u^{M} C_{a s} 9^{\circ n}$, an increase in $\mathrm{pH}^{+}$cells was observed in both the anterior and posterior regions (Figure S3C,D), suggesting that more ISCs/EBs are mitotically active.

Mutations in tumor suppressors such as Notch, neur, or Mad in progenitor cells are known to cause intestinal tumors [22,26]. To determine how effective Cas9.P2 and $u^{M} C a s 9$ are in inducing intestinal tumors, we quantified the tumor incidence rate in the population. We scored intestinal tumors as areas of dense $\mathrm{GFP}^{+}$cells containing small DAPI ${ }^{+}$nuclei (Figure 4A). Both Cas9.P2 ${ }^{\text {on } / o f f}$ and $u^{M} C_{a s} 9^{o n}$ significantly increased the percentage of tumor bearing flies when either Mad, Notch, or neur was mutated (Figure 4B), highlighting the effectiveness of these tools for tumorigenesis studies. 
A

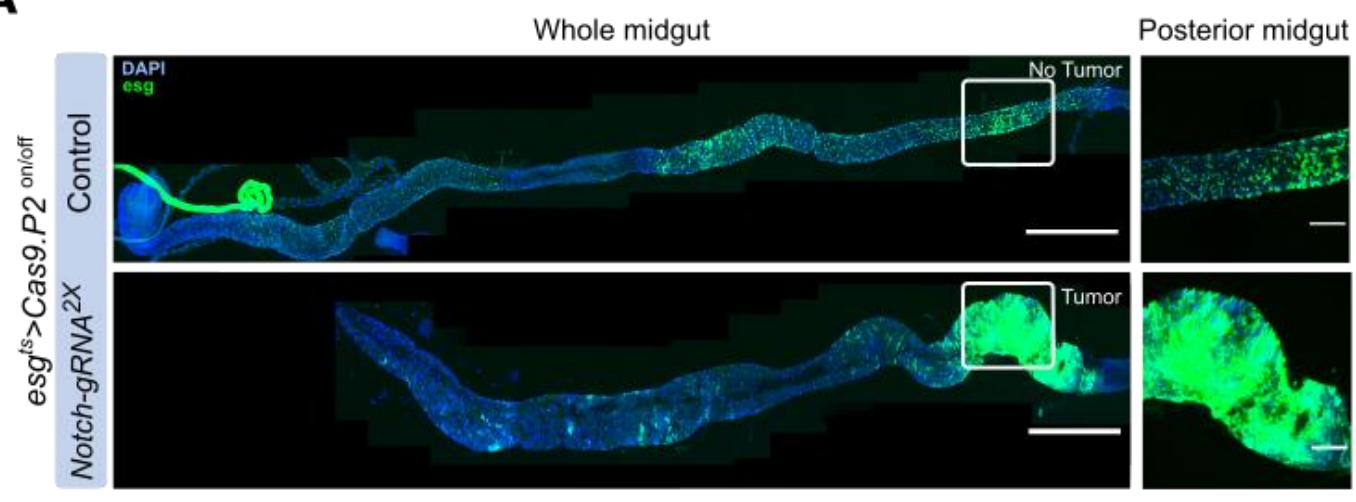

B
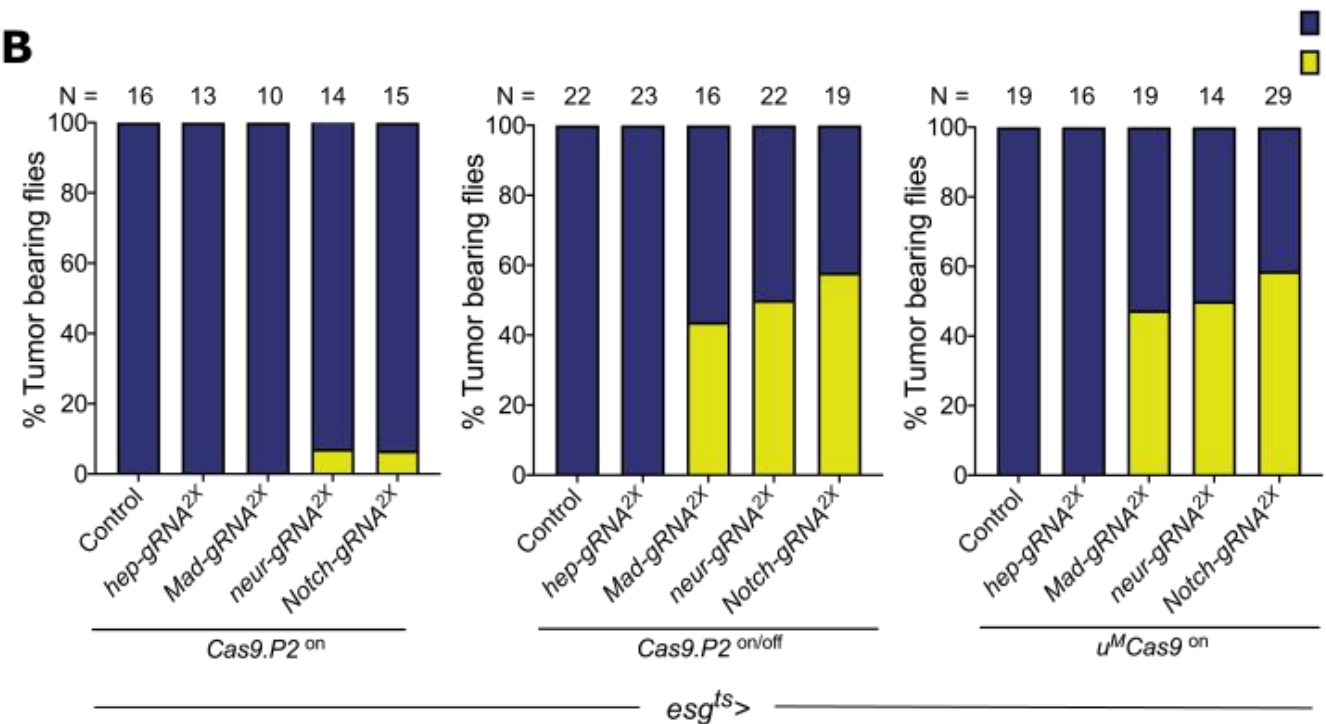

Figure 4. Tumor incidence rate increases after perturbing tumor suppressors. (A) Intestinal tumors can be observed after perturbing the Notch pathway; notice the cluster of $\mathrm{GFP}^{+}$cells accumulated in the posterior midgut (insert). (B) Quantification of the number of tumor bearing flies after perturbing the BMP, Notch, and JNK pathways. Both Cas9.P2 $2^{\text {on } / \text { ff }}$ and $u^{M} C a s g^{o n}$ increase the percentage of tumor bearing flies when $g R N A s$ were used to target Mad, neur, or Notch, while no such effects were seen with Cas9.P2 ${ }^{\text {on }}$. Scale bar: $350 \mu \mathrm{m}$, insert-60 $\mu \mathrm{m}$.

\subsection{Mutating Tumor Suppressors Changes Intestinal Morphology}

To gauge the physiological consequences of inducing intestinal tumors, we focused on morphological changes to the intestine. We quantified intestinal length after perturbing either BMP, Notch, or JNK signaling since previous studies reported that intestinal length is associated with diseases such as short bowel syndrome, which causes malnutrition, dehydration, and weight loss [28]. We observed a shortening of the intestine when either BMP (Mad $g R N A^{2 x}$ ) or Notch (Notch $g R N A^{2 x}$, neur $g R N A^{2 x}$ ) derived tumors were formed using either Cas9.P2 $2^{\text {on } / \text { off }}$ and $u^{M}$ Cas $9^{\text {on }}$ (Figure 5A,B). Moreover, Notch induced tumors also led to an accumulation of small nuclei in the epithelium (DAPI ${ }^{+}$cells), which has previously been reported to be EEs and ISCs/EBs (Figure 5A) [26]. Indeed, changes in cell composition and cell death may contribute toward the observable changes in gut length. We also observed non-significant changes in intestinal length using Cas9.P2 ${ }^{\text {on }}$ with $g R N A^{2 x}$ against hep, Mad, neur, or Notch, confirming that toxicity of Cas9.P2 ${ }^{\text {on }}$ outweighs its editing capabilities. We further quantified the diameter of the intestine in the R4 region and found that gut diameter increased when Notch signaling were perturbed using Cas9.P2 $2^{\text {on/off }}$ or $u^{M}$ Cas $9^{\text {on }}$, but not with Cas9.P2 ${ }^{\text {on }}$. In summary, we found that CRISPR induced tumorigenesis in the intestine shortens its overall length and increases the diameter of the gut, which likely impacts other physiological parameters. 
A

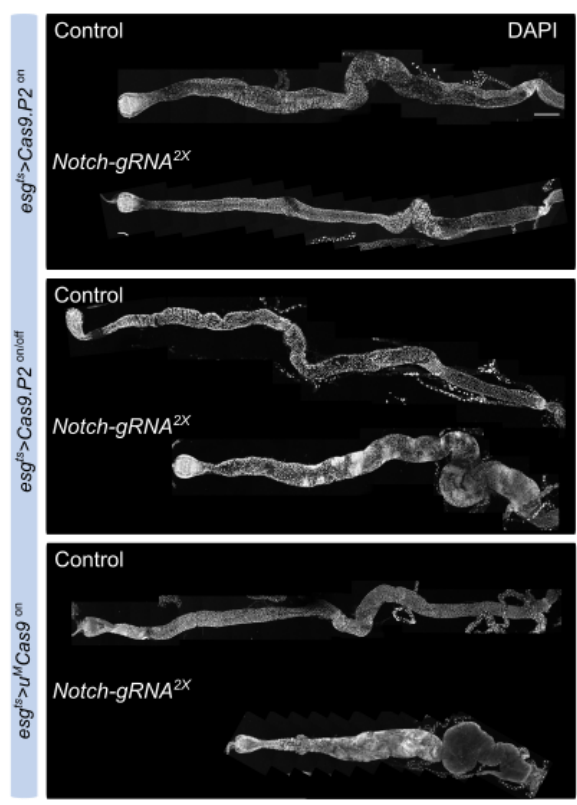

B

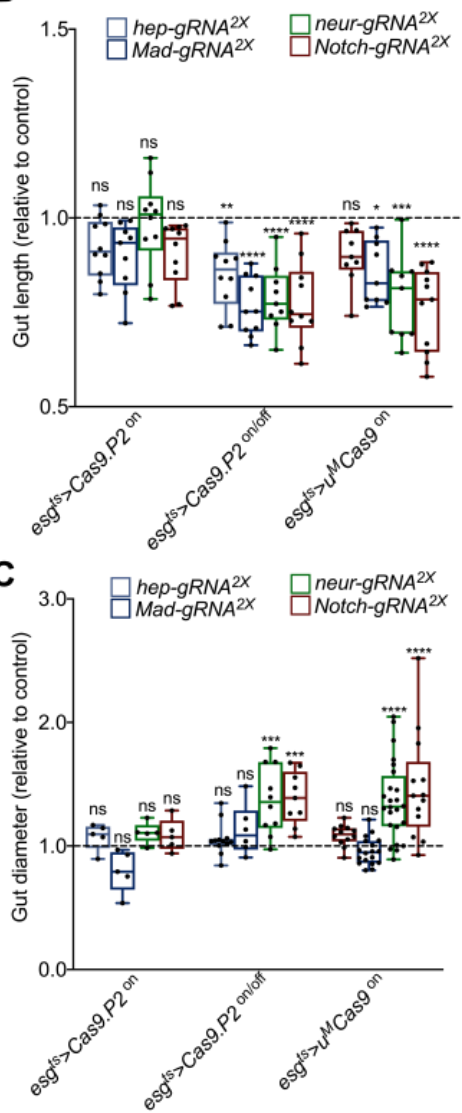

Figure 5. Intestinal morphology changes after perturbing BMP, Notch, and JNK signaling. (A) The intestine was imaged and stitched together to visualize morphological changes after perturbing the BMP, Notch, and JNK pathways. Notch perturbations are used as an example to depict intestinal length shortening. Notice clusters of DAPI ${ }^{+}$nuclei when using Cas9.P2 ${ }^{\text {on/off }}$ or $u^{M}$ Cas ${ }^{\text {on }}$ with Notch $g R N A^{2 x}$. (B) Quantification of intestinal length relative to the control. Intestinal length was shorter when the BMP, Notch, and JNK pathways were perturbed using Cas9.P2 ${ }^{\text {on } / \text { off }}$ or $u^{M}$ Cas $9^{\text {on }}$, but not with Cas9.P2 ${ }^{\text {on }}$. (C) quantification of the diameter (R4 region) of the intestine relative to the control. Gut diameter increased when Notch signaling were perturbed using Cas9.P2 ${ }^{\text {on } / \text { off }}$ or $u^{M}$ Cas $9^{\text {on }}$, but not with Cas9.P2 ${ }^{\text {on }}$. Scale bar: $350 \mu \mathrm{m} .{ }^{*} p<0.05,{ }^{* *} p<0.01,{ }^{* * *} p<0.001,{ }^{* * * *} p<0.0001$.

\section{Discussion}

Genome editing by CRISPR-Cas9 technology has greatly advanced our ability to generate mutations in vivo in a number of organisms. In the present study, we reported a simple and effective method for conditional tissue-specific CRISPR mutagenesis in the Drosophila intestine. As proof of principle, we used the Gal4/UAS system in combination with Gal80 ${ }^{\text {ts }}$ to express Cas 9 in adult esg+ intestinal progenitor cells such as ISCs and EBs, combined with two gRNAs targeting the tumor suppressors Notch, neur, and Mad. These perturbations resulted in regionalized proliferation of ISCs and tumor formation. Moreover, we showed that continuous high expression of Cas9 is deleterious to ISCs and that fine tuning its levels using different transgenes (Cas9.P2 and $u^{M}$ Cas9) or applying various temporal strategies results in mutagenesis and tumorigenesis in the intestine.

\subsection{Comparison of Intestinal-Specific CRISPR-Cas9 Systems}

RNA interference (RNAi) based LOF studies have been widely used to investigate tissue-specific functions for genes in various model organisms including Drosophila. Due to years of efforts, several in vivo RNAi transgenic libraries have been generated and used successfully in LOF studies [29-34]. These reagents have been made publicly accessible through various Drosophila stock centers (e.g., GD/KK libraries, TRiP library) $[29,35,36]$. 
However, the RNAi associated techniques have certain limitations such as off target effects and variable efficiencies.

As an alternative, CRISPR-Cas9 approaches have been applied for in vivo LOF studies. Our recently developed large-scale transgenic CRISPR-Cas9 systems, which express multiple gRNAs or genetically modified Cas9 enzymes, has greatly improved gene editing $[11,13,17]$. We previously compared RNAi and gRNA targeting Notch directly in the intestine, and showed that while Notch-RNAi generated intestinal tumors in only females, Notch-gRNA $A^{x 2}$ resulted in tumor formation in both male and female flies, suggesting that using CRISPR leads to more robust mutagenesis and intestinal tumor phenotypes [11]. The efficiency of CRISPR-Cas relies not only on the design of the gRNA and the sgRNA expression vector, but also on the extent of Cas9 expression in different tissue or cell type of interest $[11,13,17]$. Previous studies have demonstrated that CRISPR induced mutagenesis typically has efficiencies in the range of $25-100 \%$ [11,17]. Indeed, estimating the number of mutated cells in vivo using CRISPR-Cas can be cumbersome if antibodies against the targeted gene do not exist or expression of the targeted gene is not stopped. Testing the editing efficiency of the gRNAs used in this manuscript at the single cell level will be an avenue for future research.

Indeed, due to the vast array of genetic tools developed in Drosophila including different CRISPR tools, this model organism is unmatched in its ability to perform tissue-specific and cell-type specific mutagenesis. In this study, we compared the CRISPR targeting efficiency of different Cas9 transgenes and found that $u^{M} C a s 9$ yielded a stronger intestinal tumorigenic phenotype than Cas9.P2 ${ }^{\text {on }}$ when combined with $g R N A s$ against tumor suppressors. We noticed that high expression of Cas 9 protein had a negative impact on stem cell activity in the intestine, which is consistent with previous reports in other tissues or organisms $[11,37,38]$. For this reason, we developed an on/off expression strategy for Cas9.P2 by using a temperature sensitive system. As a result, Cas9.P2 ${ }^{\text {on } / \text { off }}$ has significantly enhanced intestinal tumor phenotypes when combined with gRNAs targeting the BMP and Notch pathways.

\subsection{Effects of High Cas9 Expression and Outlook on Applications of CRISPR Fly Models}

Our study demonstrates the importance of regulating Cas9 expression for effective mutagenesis. In agreement with previous studies, we showed that high expression of Cas9.P2 prevent age-associated increase in ISCs $[13,17]$. Indeed, continuous high expression of this construct also failed to recapitulate loss of function phenotypes seen when Notch and BMP signaling is perturbed in the intestine. Although we did not address how toxicity arises when Cas9.P2 is continuously expressed, we found that titrating its expression level by temporarily regulating its expression or using the genetically modified $\mathrm{u}^{\mathrm{M}}$ Cas9, results in robust tumor phenotypes developing after disrupting the Notch or BMP pathway. Future studies will be aimed at better understanding the causes of toxicity associated with high Cas9 expression.

Conserved signaling pathways such as Notch, BMP, Wnt, Ras, and JAK/STAT are essential for intestinal homeostasis and their dysregulation can result in intestinal tumorigenesis [21,25,39-46]. Nearly $75 \%$ of the genes responsible for human disease have a Drosophila homolog $[47,48]$. Recent efforts at building fly models of human cancers have allowed drug screens to take place, subsequently leading to patient studies $[46,47,49,50]$. With the precision of CRISPR-Cas in editing the genome, attempts at making patient specific mutations in fly using this strategy combined with drug screening could facilitate a more personalized approach for treatment. Additionally, with an in vivo fly model, other factors such the tumor microenvironment, host-microbe interaction, and changes in organelle/cellular trafficking pathways could also be taken into consideration in order to gain a better mechanistic understanding of tumorigenesis [21,22,51]. In summary, our study lends future support to using CRISPR-Cas editing to study tumor biology in Drosophila melanogaster, which serves as an important model organism. 
Supplementary Materials: The following are available online at https:/ /www.mdpi.com/article/ 10.3390 / cells10113156/s1, Figure S1. Boxplot representation of $\mathrm{GFP}^{+}$cells after perturbation of the BMP, Notch and JNK pathways. Figure S2. Quantification of GFP+ cells in the R5 region when Mad is perturbed with different Cas9 enzymes. Figure S3. Cas9.P2 ${ }^{\text {on } / \text { off }}$ and $\mathrm{u}^{\mathrm{M}}$ Cas $9^{\text {on }}$ mediated perturbations of tumor suppressors increase the number of mitotic cells in the intestine. Figure S4. Boxplot representation of $\mathrm{pH}^{+}$cells after perturbation of the BMP, Notch and JNK pathways.

Author Contributions: Conceptualization, S.B., S.R., J.Z., F.P. and M.B.; Formal analysis, S.B., S.R., J.Z. and T.W.; Funding acquisition, M.B. and S.R.; Investigation, S.B., S.R., J.Z., T.W. and F.P.; Supervision, S.R. and M.B.; Writing-original draft, S.R. and J.Z.; Writing-review \& editing, M.B. All authors have read and agreed to the published version of the manuscript.

Funding: Support by the SFB 873 (Deutsche Forschungsgemeinschaft), Marie Curie Fellowship (894568), and ERC Synergy DECODE (European Research Council, European Commission, 810296) is acknowledged.

Institutional Review Board Statement: Not applicable.

Informed Consent Statement: Not applicable.

Data Availability Statement: Not applicable.

Acknowledgments: We thank Flybase and the Bloomington and Vienna stock centers for fly related resources. We thank Damir Krunic, Manuela Brom, and Felix Bestvater (DKFZ imaging facility) for their assistance in image acquisition and processing.

Conflicts of Interest: The authors declare no conflict of interest.

\section{References}

1. Pickar-Oliver, A.; Gersbach, C.A. The next generation of CRISPR-Cas technologies and applications. Nat. Rev. Mol. Cell Biol. 2019, 20, 490-507. [CrossRef]

2. Barrangou, R.; Fremaux, C.; Deveau, H.; Richards, M.; Boyaval, P.; Moineau, S.; Romero, D.A.; Horvath, P. CRISPR provides acquired resistance against viruses in prokaryotes. Science 2007, 315, 1709-1712. [CrossRef] [PubMed]

3. Adli, M. The CRISPR tool kit for genome editing and beyond. Nat. Commun. 2018, 9, 1911. [CrossRef]

4. Jinek, M.; Chylinski, K.; Fonfara, I.; Hauer, M.; Doudna, J.A.; Charpentier, E. A programmable dual-RNA-guided DNA endonuclease in adaptive bacterial immunity. Science 2012, 337, 816-821. [CrossRef]

5. Chen, Y.; Wang, Z.; Ni, H.; Xu, Y.; Chen, Q.; Jiang, L. CRISPR/Cas9-mediated base-editing system efficiently generates gain-offunction mutations in Arabidopsis. Sci. China Life Sci. 2017, 60, 520-523. [CrossRef]

6. Zhan, T.; Rindtorff, N.; Betge, J.; Ebert, M.P.; Boutros, M. CRISPR/Cas9 for cancer research and therapy. Semin. Cancer Biol. 2019, 55, 106-119. [CrossRef]

7. Hales, K.G.; Korey, C.A.; Larracuente, A.M.; Roberts, D.M. Genetics on the Fly: A Primer on the Drosophila Model System. Genetics 2015, 201, 815-842. [CrossRef] [PubMed]

8. Duffy, J.B. GAL4 system in Drosophila: A fly geneticist's Swiss army knife. Genesis 2002, 34, 1-15. [CrossRef]

9. Brand, A.H.; Perrimon, N. Targeted gene expression as a means of altering cell fates and generating dominant phenotypes. Development 1993, 118, 401-415. [CrossRef] [PubMed]

10. Zeidler, M.P.; Tan, C.; Bellaiche, Y.; Cherry, S.; Hader, S.; Gayko, U.; Perrimon, N. Temperature-sensitive control of protein activity by conditionally splicing inteins. Nat. Biotechnol. 2004, 22, 871-876. [CrossRef]

11. Port, F.; Strein, C.; Stricker, M.; Rauscher, B.; Heigwer, F.; Zhou, J.; Beyersdorffer, C.; Frei, J.; Hess, A.; Kern, K.; et al. A large-scale resource for tissue-specific CRISPR mutagenesis in Drosophila. eLife 2020, 9. [CrossRef]

12. Meltzer, H.; Marom, E.; Alyagor, I.; Mayseless, O.; Berkun, V.; Segal-Gilboa, N.; Unger, T.; Luginbuhl, D.; Schuldiner, O. Tissue-specific (ts)CRISPR as an efficient strategy for in vivo screening in Drosophila. Nat. Commun. 2019, 10, 2113. [CrossRef] [PubMed]

13. Port, F.; Bullock, S.L. Augmenting CRISPR applications in Drosophila with tRNA-flanked sgRNAs. Nat. Methods 2016, 13, 852-854. [CrossRef]

14. Bier, E.; Harrison, M.M.; O'Connor-Giles, K.M.; Wildonger, J. Advances in Engineering the Fly Genome with the CRISPR-Cas System. Genetics 2018, 208, 1-18. [CrossRef] [PubMed]

15. Ren, X.; Sun, J.; Housden, B.E.; Hu, Y.; Roesel, C.; Lin, S.; Liu, L.P.; Yang, Z.; Mao, D.; Sun, L.; et al. Optimized gene editing technology for Drosophila melanogaster using germ line-specific Cas9. Proc. Natl. Acad. Sci. USA 2013, 110, 19012-19017. [CrossRef]

16. Ren, X.; Holsteens, K.; Li, H.; Sun, J.; Zhang, Y.; Liu, L.P.; Liu, Q.; Ni, J.Q. Genome editing in Drosophila melanogaster: From basic genome engineering to the multipurpose CRISPR-Cas9 system. Sci. China Life Sci. 2017, 60, 476-489. [CrossRef] 
17. Port, F.; Chen, H.M.; Lee, T.; Bullock, S.L. Optimized CRISPR/Cas tools for efficient germline and somatic genome engineering in Drosophila. Proc. Natl. Acad. Sci. USA 2014, 111, E2967-E2976. [CrossRef] [PubMed]

18. Port, F.; Muschalik, N.; Bullock, S.L. Systematic Evaluation of Drosophila CRISPR Tools Reveals Safe and Robust Alternatives to Autonomous Gene Drives in Basic Research. G3 (Bethesda) 2015, 5, 1493-1502. [CrossRef]

19. Miguel-Aliaga, I.; Jasper, H.; Lemaitre, B. Anatomy and Physiology of the Digestive Tract of Drosophila melanogaster. Genetics 2018, 210, 357-396. [CrossRef]

20. Liu, Q.; Jin, L.H. Tissue-resident stem cell activity: A view from the adult Drosophila gastrointestinal tract. Cell Commun. Signal. 2017, 15, 33. [CrossRef]

21. Zhou, J.; Boutros, M. JNK-dependent intestinal barrier failure disrupts host-microbe homeostasis during tumorigenesis. Proc. Natl. Acad. Sci. USA 2020, 117, 9401-9412. [CrossRef]

22. Zhou, J.; Valentini, E.; Boutros, M. Microenvironmental innate immune signaling and cell mechanical responses promote tumor growth. Dev. Cell 2021, 56, 1884-1899 e1885. [CrossRef] [PubMed]

23. Jiang, H.; Patel, P.H.; Kohlmaier, A.; Grenley, M.O.; McEwen, D.G.; Edgar, B.A. Cytokine/Jak/Stat signaling mediates regeneration and homeostasis in the Drosophila midgut. Cell 2009, 137, 1343-1355. [CrossRef]

24. Schindelin, J.; Arganda-Carreras, I.; Frise, E.; Kaynig, V.; Longair, M.; Pietzsch, T.; Preibisch, S.; Rueden, C.; Saalfeld, S.; Schmid, B.; et al. Fiji: An open-source platform for biological-image analysis. Nat. Methods 2012, 9, 676-682. [CrossRef]

25. Biteau, B.; Hochmuth, C.E.; Jasper, H. JNK activity in somatic stem cells causes loss of tissue homeostasis in the aging Drosophila gut. Cell Stem Cell 2008, 3, 442-455. [CrossRef]

26. Patel, P.H.; Dutta, D.; Edgar, B.A. Niche appropriation by Drosophila intestinal stem cell tumours. Nat. Cell Biol. 2015, 17, 1182-1192. [CrossRef] [PubMed]

27. Guo, Z.; Driver, I.; Ohlstein, B. Injury-induced BMP signaling negatively regulates Drosophila midgut homeostasis. J. Cell Biol. 2013, 201, 945-961. [CrossRef] [PubMed]

28. Massironi, S.; Cavalcoli, F.; Rausa, E.; Invernizzi, P.; Braga, M.; Vecchi, M. Understanding short bowel syndrome: Current status and future perspectives. Dig. Liver Dis. 2020, 52, 253-261. [CrossRef]

29. Dietzl, G.; Chen, D.; Schnorrer, F.; Su, K.C.; Barinova, Y.; Fellner, M.; Gasser, B.; Kinsey, K.; Oppel, S.; Scheiblauer, S.; et al. A genome-wide transgenic RNAi library for conditional gene inactivation in Drosophila. Nature 2007, 448, 151-156. [CrossRef]

30. Horn, T.; Arziman, Z.; Berger, J.; Boutros, M. GenomeRNAi: A database for cell-based RNAi phenotypes. Nucleic Acids Res. 2007, 35, D492-D497. [CrossRef]

31. Horn, T.; Boutros, M. E-RNAi: A web application for the multi-species design of RNAi reagents-2010 update. Nucleic Acids Res. 2010, 38, W332-W339. [CrossRef] [PubMed]

32. Ni, J.Q.; Liu, L.P.; Binari, R.; Hardy, R.; Shim, H.S.; Cavallaro, A.; Booker, M.; Pfeiffer, B.D.; Markstein, M.; Wang, H.; et al. A Drosophila resource of transgenic RNAi lines for neurogenetics. Genetics 2009, 182, 1089-1100. [CrossRef]

33. Ni, J.Q.; Markstein, M.; Binari, R.; Pfeiffer, B.; Liu, L.P.; Villalta, C.; Booker, M.; Perkins, L.; Perrimon, N. Vector and parameters for targeted transgenic RNA interference in Drosophila melanogaster. Nat. Methods 2008, 5, 49-51. [CrossRef]

34. Green, E.W.; Fedele, G.; Giorgini, F.; Kyriacou, C.P. A Drosophila RNAi collection is subject to dominant phenotypic effects. Nat. Methods 2014, 11, 222-223. [CrossRef] [PubMed]

35. Larkin, A.; Marygold, S.J.; Antonazzo, G.; Attrill, H.; Dos Santos, G.; Garapati, P.V.; Goodman, J.L.; Gramates, L.S.; Millburn, G.; Strelets, V.B.; et al. FlyBase: Updates to the Drosophila melanogaster knowledge base. Nucleic Acids Res. 2021, 49, D899-D907. [CrossRef]

36. Whitworth, C. The Bloomington Drosophila Stock Center: Management, Maintenance, Distribution, and Research. In The Biological Resources of Model Organisms; Jarret, R.L., McCluskey, K., Eds.; Taylor \& Francis Group: London, UK, 2019 ; pp. 145-162.

37. Jiang, W.; Brueggeman, A.J.; Horken, K.M.; Plucinak, T.M.; Weeks, D.P. Successful transient expression of Cas 9 and single guide RNA genes in Chlamydomonas reinhardtii. Eukaryot. Cell 2014, 13, 1465-1469. [CrossRef] [PubMed]

38. Yang, S.; Li, S.; Li, X.J. Shortening the Half-Life of Cas9 Maintains Its Gene Editing Ability and Reduces Neuronal Toxicity. Cell Rep. 2018, 25, 2653-2659 e2653. [CrossRef]

39. Wang, C.; Zhao, R.; Huang, P.; Yang, F.; Quan, Z.; Xu, N.; Xi, R. APC loss-induced intestinal tumorigenesis in Drosophila: Roles of Ras in Wnt signaling activation and tumor progression. Dev. Biol. 2013, 378, 122-140. [CrossRef]

40. Ferguson, M.; Petkau, K.; Shin, M.; Galenza, A.; Fast, D.; Foley, E. Differential effects of commensal bacteria on progenitor cell adhesion, division symmetry and tumorigenesis in the Drosophila intestine. Development 2021, 148. [CrossRef]

41. Suijkerbuijk, S.J.; Kolahgar, G.; Kucinski, I.; Piddini, E. Cell Competition Drives the Growth of Intestinal Adenomas in Drosophila. Curr. Biol. 2016, 26, 428-438. [CrossRef]

42. Markstein, M.; Dettorre, S.; Cho, J.; Neumuller, R.A.; Craig-Muller, S.; Perrimon, N. Systematic screen of chemotherapeutics in Drosophila stem cell tumors. Proc. Natl. Acad. Sci. USA 2014, 111, 4530-4535. [CrossRef] [PubMed]

43. Ngo, S.; Liang, J.; Su, Y.H.; O’Brien, L.E. Disruption of EGF Feedback by Intestinal Tumors and Neighboring Cells in Drosophila. Curr. Biol. 2020, 30, 1537-1546 e1533. [CrossRef]

44. Cordero, J.B.; Stefanatos, R.K.; Myant, K.; Vidal, M.; Sansom, O.J. Non-autonomous crosstalk between the Jak/Stat and Egfr pathways mediates Apc1-driven intestinal stem cell hyperplasia in the Drosophila adult midgut. Development 2012, 139, 4524-4535. [CrossRef] 
45. Zhai, W.; Lim, T.K.; Zhang, T.; Phang, S.T.; Tiang, Z.; Guan, P.; Ng, M.H.; Lim, J.Q.; Yao, F.; Li, Z.; et al. The spatial organization of intra-tumour heterogeneity and evolutionary trajectories of metastases in hepatocellular carcinoma. Nat. Commun. 2017, 8, 4565. [CrossRef]

46. Bilder, D.; Ong, K.; Hsi, T.C.; Adiga, K.; Kim, J. Tumour-host interactions through the lens of Drosophila. Nat. Rev. Cancer 2021, 21, 687-700. [CrossRef] [PubMed]

47. Pandey, U.B.; Nichols, C.D. Human disease models in Drosophila melanogaster and the role of the fly in therapeutic drug discovery. Pharm. Rev. 2011, 63, 411-436. [CrossRef]

48. Ugur, B.; Chen, K.; Bellen, H.J. Drosophila tools and assays for the study of human diseases. Dis. Model. Mech. 2016, 9, 235-244. [CrossRef]

49. Bangi, E.; Murgia, C.; Teague, A.G.; Sansom, O.J.; Cagan, R.L. Functional exploration of colorectal cancer genomes using Drosophila. Nat. Commun. 2016, 7, 13615. [CrossRef] [PubMed]

50. Bangi, E.; Ang, C.; Smibert, P.; Uzilov, A.V.; Teague, A.G.; Antipin, Y.; Chen, R.; Hecht, C.; Gruszczynski, N.; Yon, W.J.; et al. A personalized platform identifies trametinib plus zoledronate for a patient with KRAS-mutant metastatic colorectal cancer. Sci. Adv. 2019, 5, eaav6528. [CrossRef]

51. Redhai, S.; Boutros, M. The Role of Organelles in Intestinal Function, Physiology, and Disease. Trends Cell Biol. 2021, 31, 485-499. [CrossRef] 